\title{
Barriers and facilitators to taking folic acid supplements among women aged 18-30 years
}

\author{
M. F. Faughnan, D. Cahill, L. N. Burgoyne and I. J. Perry \\ ${ }^{1}$ Safefood, Eastgate Avenue, Little Island, Cork, Republic of Ireland, ${ }^{2}$ Health Promotion Department, \\ Health Service Executive South, Cork, Republic of Ireland and ${ }^{3}$ Department of Epidemiology and Public Health, \\ Brookfield Health Sciences Complex, University College Cork, Cork, Republic of Ireland
}

Although awareness of folic acid is high among women of child-bearing age in Ireland only approximately one-quarter of women report taking folic acid peri-conceptually ${ }^{(1)}$. The current research aimed to explore the barriers and motivators to taking folic acid supplements among women aged 18-30 years across socio-economic status.

Data were collected in six focus groups that included a total of thirty-five women and were held in the city and suburban areas of Cork in the Republic of Ireland, three in areas designated as disadvantaged ( $n$ 18) and three in non-disadvantaged areas ( $n$ 17). The data were analysed using a grounded theory approach.

Five major themes were identified in relation to barriers and facilitators to taking folic acid across all groups (Table). The most pertinent social-class difference that was evident was scepticism and a fatalistic view of spina bifida that existed among participants in disadvantaged areas.

Table. Major themes that emerged during focus groups and illustrative quotes

\begin{tabular}{|c|c|}
\hline Theme & Illustrative quotations from focus group discussions \\
\hline $\begin{array}{l}\text { Association of folic acid } \\
\text { with pregnancy }\end{array}$ & 'You wouldn't take it like if you weren't planning on getting pregnant. It's like half admitting you might get' \\
\hline Perception of harm & 'If you took it, for no reason for six years and you didn't have a baby, would you get an overload of it?' \\
\hline Attitudes of significant others & $\begin{array}{l}\text { 'I even know my own mother you know, you mentioned she'd like I had five children and didn't do this didn't do } \\
\text { that and ye were all grand' }\end{array}$ \\
\hline Perceived importance of the folic acid & 'A lot of people still don't know so they should really make it talked about like everywhere' \\
\hline Cost $v$. benefit analysis & 'It's passing through you like if you're not pregnant and doesn't do anything for you what's the point in taking it' \\
\hline
\end{tabular}

Participants consistently cited that their only association with folic acid was with pregnancy. Planning or being currently pregnant was viewed as a strong motivator. In contrast, a successful previous pregnancy without taking folic acid and not planning a pregnancy emerged as strong barriers.

Concern of overdosing with folic acid emerged in all groups. Discomfort in swallowing tablets and the fear of side effects such as nausea were the prominent fears for disadvantaged groups whereas the artificial nature of taking a supplement emerged in non disadvantaged groups.

The attitudes of significant others was viewed as either a motivator or a barrier. If taking vitamin supplements was not typical behaviour among family and friends this factor presented a major barrier to taking folic acid.

A perceived low visibility of the folic acid recommendation in the mass media and among health professionals diminished the importance of the issue for participants. Hearing the recommendation from a trusted health professional was viewed as facilitating, e.g. in association with receiving contraception. Fear of spina bifida was seen as a facilitating factor for taking folic acid and participants viewed the mass media as a vehicle for this factor.

The effort, i.e. personal cost, required to build folic acid into everyday routines outweighed the benefit to a potential pregnancy in the future for most participants. They acknowledged a preference for more immediate benefits. Cost was highlighted as a minor barrier.

The findings indicate that health promotion initiatives at both a population and an individual level are required to generate sufficient visibility of folic acid advice for it to be perceived by the target audience as important. Knowledge of the barriers and motivators to folic acid supplement use is essential to underpin all health promotion initiatives.

This research was supported by safefood and Population Health, Health Service Executive.

1. Food Safety Authority of Ireland (2006) Report of the National Committee on Folic Acid Food Fortification. http://www.fsai.ie/uploadedFiles/folic acid.pdf 